\title{
Effect of in situ water content variation on the spatial variation of strength of deep cement-mixed clay
}

\author{
Y. LIU*, L. Q. HE†, Y. J. JIANG屯, M. M. SUN§, E. J. CHEN\| and F.-H. LEE†
}

\begin{abstract}
This paper examines the interaction between the spatial variations in binder concentration (i.e. cement slurry concentration) and in situ water content, in cement-mixed soil, using field and model data as well as statistical analysis and random field simulation. The field data are first analysed to shed light on the spatial variation in the in situ water content, including its scale of fluctuation. A statistical model is then developed which takes into account the variation in binder concentration and in situ water content. This leads to a two-parameter model for the prediction of the mean, variance and probability distribution function of the strength of the cement-treated soil. The scale of fluctuation for the variation in binder concentration arising from imperfect mixing within a cement-mixed column is then examined using centrifuge model data. This indicates that the scale of fluctuation in binder concentration is much shorter in range than that of the in situ water content. The combined effect of these two scales of fluctuation is then studied by simulating the resulting random field using Monte-Carlo simulations. This indicates that the size of the sampling region has a significant effect on the scale of fluctuation that is captured. If the sampling region is of a similar size to the column diameter, the measured scale of fluctuation reflects that of the binder concentration. As the size of the sampling region increases, so does the measured scale of fluctuation. This explains the wide range of scales of fluctuation that have been reported for cementtreated soil. To capture both scales of fluctuation in core sampling, some boreholes should be sunk at close spacings of less than a column diameter, in order to capture short-range variation.
\end{abstract}

KEYWORDS: centrifuge modelling; ground improvement; grouting; soil stabilisation; statistical analysis

\section{INTRODUCTION}

Deep cement mixing is often used for improvement of soft clay in underground constructions. The strength of cementadmixed ground often exhibits significant spatial variation (e.g. Bruce et al., 2013; Namikawa, 2015; Tang et al., 2015). For instance, Bruce et al. (2013) observed that the strength of deep-mixed ground is about twice as variable as the strength of natural clay deposits. However, there is significant difference between the results from different sites and different studies into such spatial variability (e.g. Honjo, 1982; Larsson et al., 2005; Navin, 2005; Lee et al., 2006, 2008; Larsson \& Nilsson, 2009; Filz \& Navin, 2010; Chen et al., 2016). For instance, Honjo (1982) reported a coefficient of variation $(\mathrm{CoV})$ ranging from 0.3 to 0.6 for different sites in Japan. As Table 1 shows, the scale of fluctuation (SoF) values are equally, if not more, variable, and they range from less than a column diameter to several column diameters. An SoF of several column diameters would imply that the

Manuscript received 7 June 2017; revised manuscript accepted 10 May 2018. Published online ahead of print 8 June 2018.

Discussion on this paper closes on 1 October 2019, for further details see p. ii.

Published with permission by the ICE under the CC-BY 4.0 license. (http://creativecommons.org/licenses/by/4.0/)

* State Key Laboratory of Water Resources and Hydropower Engineering Science, Wuhan University, Wuhan, P. R. China.

$\dagger$ Department of Civil and Environmental Engineering, National University of Singapore, Singapore.

\$ Ed Zublin AG, Singapore Branch - Pipejacking Division, Singapore.

$\S$ Department of Civil Engineering, Zhejiang University City College, Hangzhou, P. R. China.

\| School of Civil Engineering and Mechanics, Huazhong University of Science and Technology, Wuhan, P. R. China (corresponding author). strength within a column should be fairly uniform. This would contradict the data of Larsson et al. (2005) and Chen et al. (2016), which indicate that there is significant variability within a deep-mixed column, arising from imperfect mixing of the cement binder and the soil.

The causes of these discrepancies remain unclear at present. However, to the extent that cement-treated soil is created from natural soil by man-made processes, it may possess both man-made and natural variability. The former is typified by the variation in binder concentration (i.e. cement slurry concentration) arising from imperfect mixing. Chen et al. (2016) showed that variation in binder concentration can explain much of the observed core strength distribution from field data. Although Chen et al. (2016) did not consider the SoF, it is reasonable to surmise that the horizontal SoF arising from imperfect mixing should be less than one column diameter, since the mixing is done column by column and each column is independent of the others. This would contradict field observations that horizontal SoF can span across several column diameters.

One possible reason for the greater range of SoF is natural variability in soil properties (e.g. Phoon \& Kulhawy, 1999; Li et al., 2016). However, as deep mixing involves breaking up of the in situ soil structure, the latter is unlikely to have an important effect on the cement-treated soil strength. Other properties include those related to soil type and mineralogy, such as Atterberg limits, as well as state variables such as water content and undrained shear strength. Data on spatial variation in mineralogy and Atterberg limits within a single soil formation remain scarce to date. De Souza et al. (2009) presented data on variation in kaolinite and gibbsite content in near-surface soil down to a depth of about $0.8 \mathrm{~m}$ in a sloping landform measuring $100 \mathrm{~m}$ by $200 \mathrm{~m}$, but this is likely to be strongly influenced by vegetation and weathering. Hence, it may not reflect mineralogical variation at larger depths. Moreover, the effect of Atterberg limits on the 
Table 1. Summary of scales of fluctuation

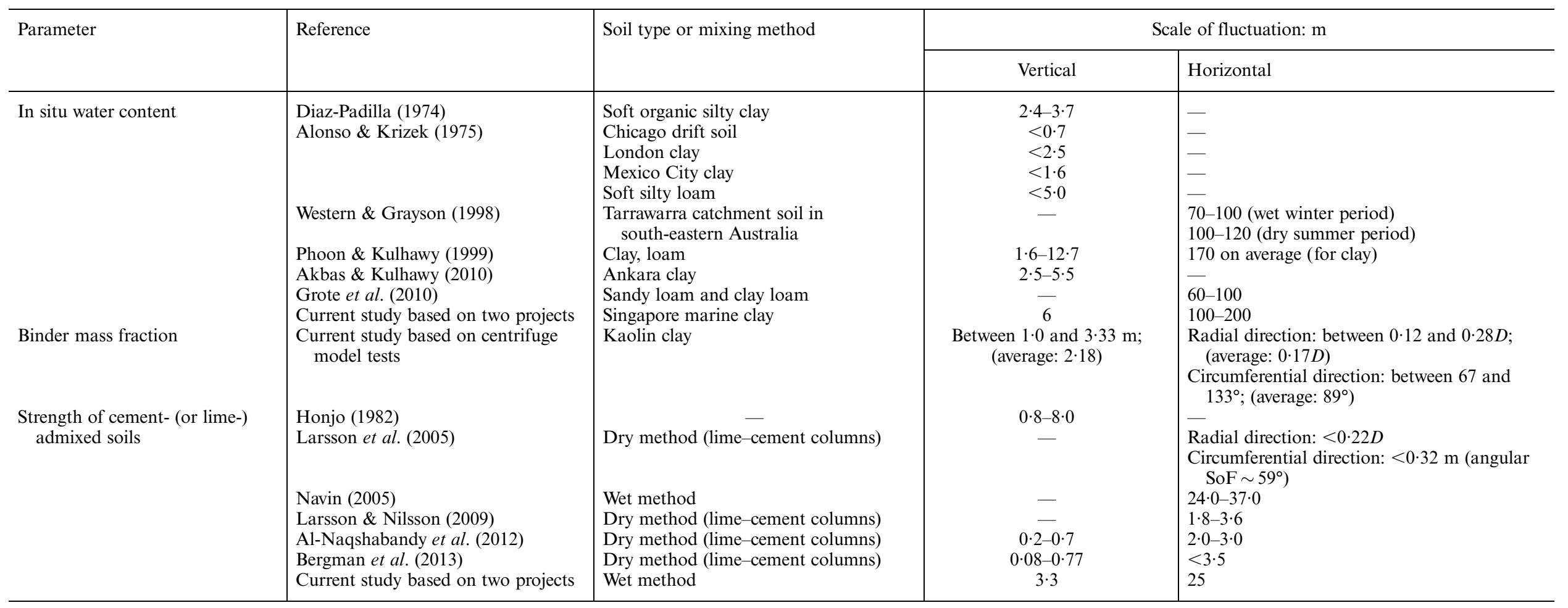

Note: $D=$ diameter of column, which is $2.4 \mathrm{~m}$ (prototype) in centrifuge model in this study. 


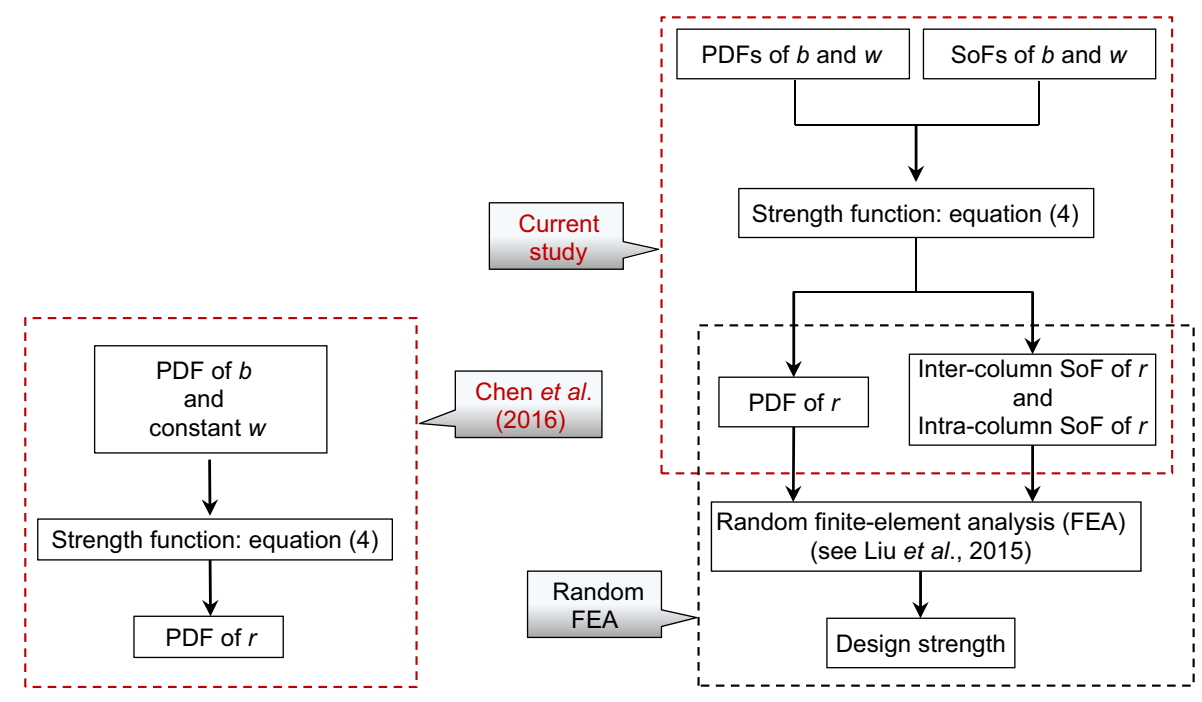

(a)

(b)

Fig. 1. Structure and flow chart of (a) the Chen et al. (2016) study and (b) the current study (notation as defined in text and the notation list)

strength of cement-admixed soil is still not well-understood. Thus, variations in Atterberg limits cannot be examined. The in situ soils are disturbed during the mixing process, and thus the influence of overconsolidation ratio (OCR) is weakened, which will be shown by the centrifuge model tests. For this reason, the effect of OCR is also excluded. As a composite material, the cement-clay admixture consists of three components - namely, cement, soil and water. In this regard, it would not be unreasonable to examine the statistical characteristics of the admixture from the fractions of those three components.

It is well recognised that, for the same soil, the undrained shear strength decreases as its water content increases. Chen et al. (2016) reported significant variation in the in situ water content in the Marina Bay financial centre (MBFC) site. However, their model was unable to consider the spatial variation in the in situ water content. To assess the possible effect of in situ water content on the strength of the cementadmixed soil, they conducted a sensitivity study, which indicated that the in situ water content had a measurable effect on the strength distribution of the cement-treated clay through the total water content of the cement-clay admixture. The effect of in situ water content on the strength of cement-clay admixture has also been discussed by Tsuchida \& Tang (2015), in a deterministic manner.

This paper examines the effect of in situ water content on two aspects of spatial variation in the strength of cementtreated clay, namely, the probability distribution, especially the mean and CoV, as well as the SoF. In situ water content variations in the MBFC and Marina One (Liu et al., 2017) sites are first characterised. The model by Chen et al. (2016) is then extended to include the natural water content as a second random variable, as shown in Fig. 1. This leads to a strength distribution relation for the unconfined compression strength of cement-admixed soil, which is a function of cement binder concentration and in situ water content. The SoF is then studied numerically by repeated realisations of the deep-mixed soil mass, taking into account the stochastic variation in binder concentration, positioning error of columns and the SoF of the in situ water content. The positioning error of columns refers to the deviation of column position from its designated position, which depends on the drilling verticality and is proportional to the depth of the improved zone from the ground level (see Liu et al., 2015). The relationship among the variances of strength ratio, in situ water content and binder mass fraction is explored. Finally, the computed statistics are compared with those of the measured and previously reported data.

\section{SITE CONDITIONS}

The site conditions for the MBFC (Chen et al., 2016) and Marina One (Liu et al., 2017) have already been reported previously and will not be repeated herein. Cement treatment was implemented on the two marine clay layers in Fig. 2. In general, the lower marine clay layer has lower water content than the upper marine clay. Within the upper marine clay layer, the water content seems to be slightly higher at the mid-layer, although there is significant scatter. This may be attributed to the fact that both sites are on reclaimed land and the upper marine clay may still be consolidating under the sand fill, which implies that the soils of both sites are normally consolidated. However, the average in situ water content for each depth of all the three layers shows a decreasing trend with depth, Fig. 3(a). This correlates inversely with the unconfined compressive strength of the cement-admixed soil, which shows an increasing trend, Fig. 3(b). The spatial variation of water content and the differences in horizontal and vertical directions may be because the drainage paths of these two sites are uncertain but generally vertical and layered. The effect of these statistical characteristics on the cemented material will be discussed in detail in this study. As Table 2 shows, the in situ water content distribution passed the Kolmogorov-Smirnov goodness-of-fit test for the normal distribution.

As shown in Figs 4(a)-4(c), using an exponential autocorrelation function led to an SoF of in situ water content ranging from $100 \mathrm{~m}$ to $200 \mathrm{~m}$ in the horizontal plane, and approximately $6 \mathrm{~m}$ in the vertical direction. The spatial variation of water content and the differences in horizontal and vertical directions may be because the drainage paths of these two sites are uncertain but generally vertical and layered. These findings are generally consistent with those in Phoon \& Kulhawy (1999), where the average SoF of in situ water content was found to be $170 \mathrm{~m}$ and $5.7 \mathrm{~m}$ in the horizontal and vertical directions, respectively. For the unconfined compressive strength of the cement-admixed soil, Fig. 5 suggests a vertical SoF of $3 \cdot 3 \mathrm{~m}$. This falls within the range of $0.8 \mathrm{~m}$ to $8 \mathrm{~m}$ reported by Honjo (1982). The horizontal SoF was estimated to be $25 \mathrm{~m}$; this lies within the range of $24 \mathrm{~m}$ to 


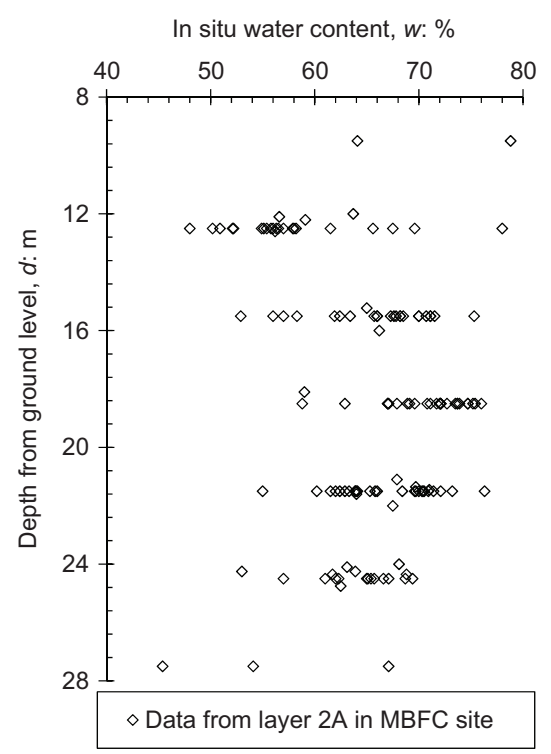

(a)

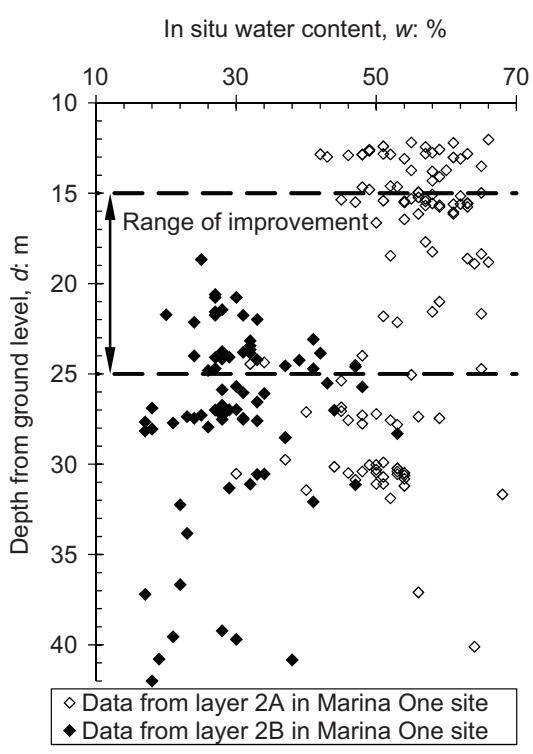

(b)

Fig. 2. Data points of in situ water content from: (a) MBFC site; (b) Marina One site (layer 2A: upper marine clay; layer 2B: lower marine clay)

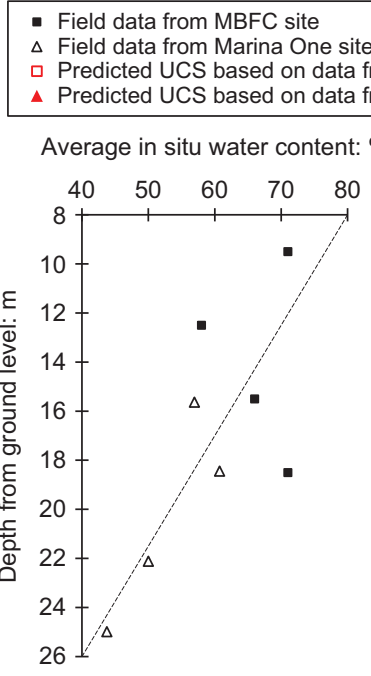

(a) ne site

Average UCS: MPa

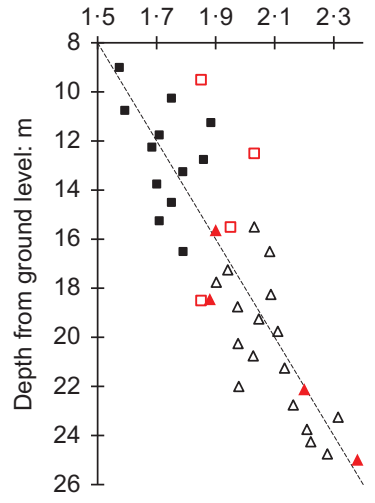

(b)
Fig. 3. Linear correlation between: (a) in situ water content averaged within $2 \mathrm{~m}$; (b) measured and predicted unconfined compressive strength (UCS)

$37 \mathrm{~m}$ reported by Navin (2005). Table 1 indicates that the SoF is often estimated as a range rather than a single value. This is because the determination of an exact value of SoF, as a second-order statistic, requires a large volume of data, which are often not available. In this study, the data points for determining SoFs are provided and a representative SoF value is roughly estimated. An interval for the corresponding SoF can also be interpreted for other purposes.

\section{PROBABILITY DISTRIBUTION FUNCTION}

Two methods for determining the strength probability distribution function (PDF) were developed. In the simplified semi-theoretical method, the mean and variance were first estimated. This allows an appropriate and commonly used PDF to be selected to fit the estimated mean and variance. The model by Chen et al. (2016) is based on a modified form of the empirical relation between the unconfined compressive strength (UCS), $q_{\mathrm{u}}$ and the mix ratio of cement-treated soil in Xiao et al. (2014), which is given by

$$
r=\frac{q_{\mathrm{u}}}{q_{0}}=\frac{1+m x+(m x)^{2}}{y^{n}}
$$

where $r$ is the normalised strength; $m$ and $n$ are fitted constants, and they can be taken as 0.28 and 2.93 , respectively, following Xiao et al. (2014); $q_{0}$ reflects the effect of curing time and strength gain ratio, as extensively discussed by Xiao et al. (2014). In equation (1), $x$ and $y$ are the soilcement and water-cement mass ratios, respectively, in the cement-admixed soil at the point of mixing. These ratios can be related to the water-cement mass ratio $a$ of the cement slurry, binder mass fraction in the mixed soil $b$ and in situ water content $w$ of the soft clay by (see details of derivation in Chen et al. (2016))

$$
\begin{aligned}
& x=\frac{1+a}{1+w}\left(\frac{1}{b}-1\right) \\
& y=w \times x+a
\end{aligned}
$$

The definitions of $a, b$ and $w$ in terms of phase relationship are depicted in Fig. 6. As discussed earlier, the variables $b$ and $w$ are regarded as random variables herein. Equation (1) can be rewritten as a general function of $b$ and $w$

$$
r=r(b, w)
$$

By applying equations (1)-(3) to the average in situ water content in Fig. 3(a), the corresponding unconfined compressive strength can be determined, as shown in Fig. 3(b). These also roughly follow the same trend as the average unconfined compressive strength of the treated soil in the same figure, indicating the increase in average unconfined compressive strength in Fig. 3(b) can be largely attributed to the decrease in the in situ water content with depth.

The mean and variance of the normalised strength can be estimated approximately through Taylor expansion on equation (4) and ignoring higher order terms, as

$$
\bar{r}=r(\bar{b}, \bar{w})+\frac{\operatorname{Var}[b]}{2} \times V_{1}+\frac{\operatorname{Var}[w]}{2} \times V_{2}
$$


Table 2. Statistical characteristics of in situ water content, $w$

\begin{tabular}{|c|c|c|c|c|}
\hline \multirow[t]{2}{*}{ Soil range } & \multirow[t]{2}{*}{ Parameter } & \multirow{2}{*}{$\frac{\mathrm{MBFC}}{\text { Layer 2A }}$} & \multicolumn{2}{|c|}{ Marina One } \\
\hline & & & Layer 2A & Layer 2B \\
\hline \multirow{5}{*}{ Within range of improvement } & Sample size & 83 & 42 & 29 \\
\hline & Depth range: $\mathrm{m}$ & $10-20$ & $15-25$ & $15-25$ \\
\hline & Mean & $69 \cdot 1 \%$ & $56 \%$ & $31 \%$ \\
\hline & $\mathrm{CoV}$ & $0 \cdot 08$ & $0 \cdot 13$ & $0 \cdot 20$ \\
\hline & Maximum value & $78 \%$ & $66 \%$ & $47 \%$ \\
\hline \multirow[t]{9}{*}{ Whole depth } & Sample size & 134 & 91 & 122 \\
\hline & Depth range: $\mathrm{m}$ & $9-30$ & $12-40$ & $18-48$ \\
\hline & Mean & $65 \%$ & $54 \%$ & $29 \%$ \\
\hline & $\mathrm{CoV}$ & $0 \cdot 11$ & $0 \cdot 14$ & $0 \cdot 06$ \\
\hline & Minimum value & $45 \%$ & $30 \%$ & $15 \%$ \\
\hline & Maximum value & $80 \%$ & $69 \%$ & $53 \%$ \\
\hline & KS test for normality & $D_{0}=0 \cdot 078$ & $D_{0}=0.060$ & $D_{0}=0 \cdot 114$ \\
\hline & Horizontal SoF: m & 200 & 100 & 100 \\
\hline & Vertical SoF: m & 6 & 6 & 6 \\
\hline
\end{tabular}

Note: $\mathrm{KS}=$ Kolmogorov-Smirnov; $D_{0}=$ maximum vertical distance between empirical and fitted CDFs. The critical $D_{0}$ values are calculated at significance level 0.05. (Layer 2A: upper marine clay; layer 2B: lower marine clay.)

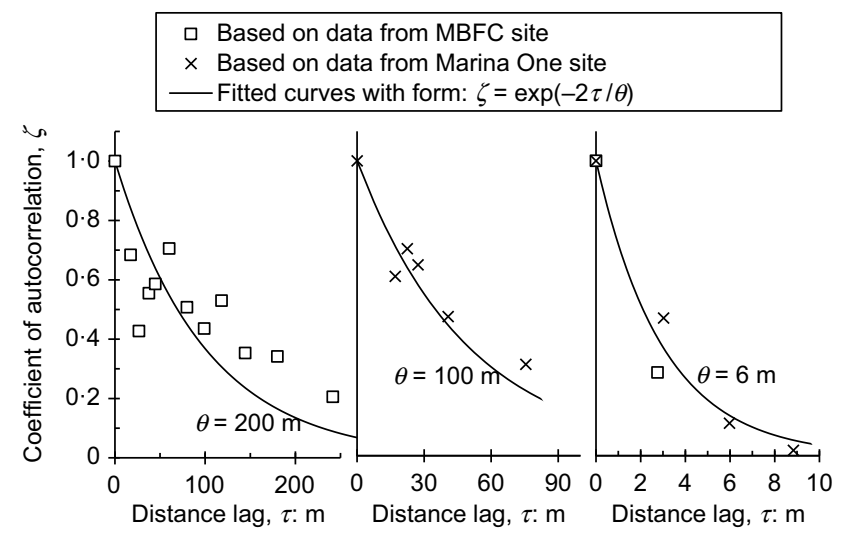

(a)

(b)

(c)

Fig. 4. Autocorrelation functions of in situ water content $(\theta$ : scale of fluctuation): (a), (b) horizontal plane; (c) vertical direction

$$
\begin{aligned}
& \operatorname{Var}[r]=\operatorname{Var}[b] \times V_{3}+\operatorname{Var}[w] \times V_{4} \\
& \operatorname{CoV}[r]=\frac{1}{\bar{r}} \sqrt{V_{3}(\bar{b} \times \operatorname{CoV}[b])^{2}+V_{4}(\bar{w} \times \operatorname{CoV}[w])^{2}}
\end{aligned}
$$

in which symbols with a bar denote their mean values; Var[-] and $\mathrm{CoV}[-]$ denote the variance and $\mathrm{CoV}$, respectively; and $V_{1}, V_{2}, V_{3}$ and $V_{4}$ are variables associated with the partial derivatives of equation (4) as derived in Appendix 1. Fig. 7 tabulates the values of $r, V_{1}, V_{2}, V_{3}$ and $V_{4}$ to facilitate the estimation of mean and variance of the strength ratio by equations (5) and (6). As $V_{1}$ and $V_{2}$ are both positive in the possible ranges of mean binder mass fraction and in situ water content, equation (5) indicates that $\bar{r}>r(\bar{b}, \bar{w})$; in other words, by directly substituting average binder mass fraction and in situ water content into the strength function, it is possible to obtain a safe estimate of the mean strength.

Based on the mean and variance from equations (5) and (6), a PDF (e.g. lognormal distribution) can be fitted. The lower bound strength of the distribution can be evaluated using the maximum in situ water content and minimum binder mass fraction in equation (1). Likewise, the upper bound of strength ratio can also be calculated. This allows a PDF with bounds (e.g. beta distribution) to be fitted.

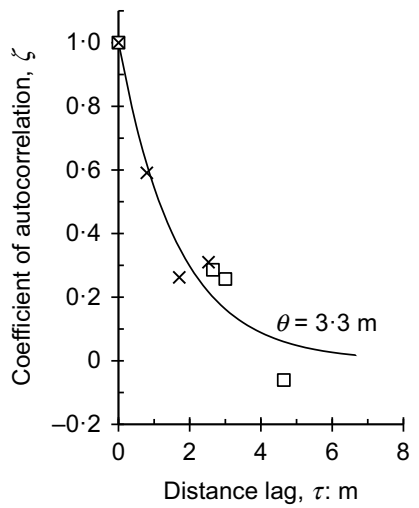

(a)

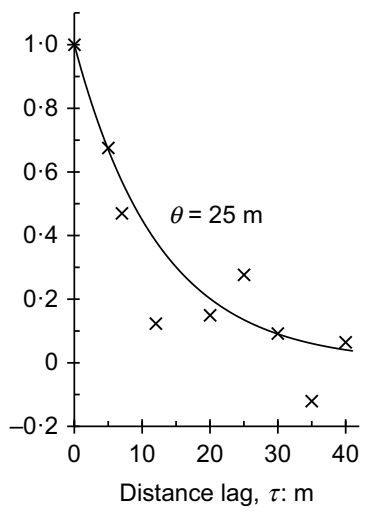

(b)
Fig. 5. Autocorrelation function of unconfined compressive strength of cement-admixed soils based on field data. The horizontal SoF could not be determined from the MBFC core strength data as the northings and eastings of the cores were unavailable (key as per Fig. 4): (a) vertical; (b) horizontal

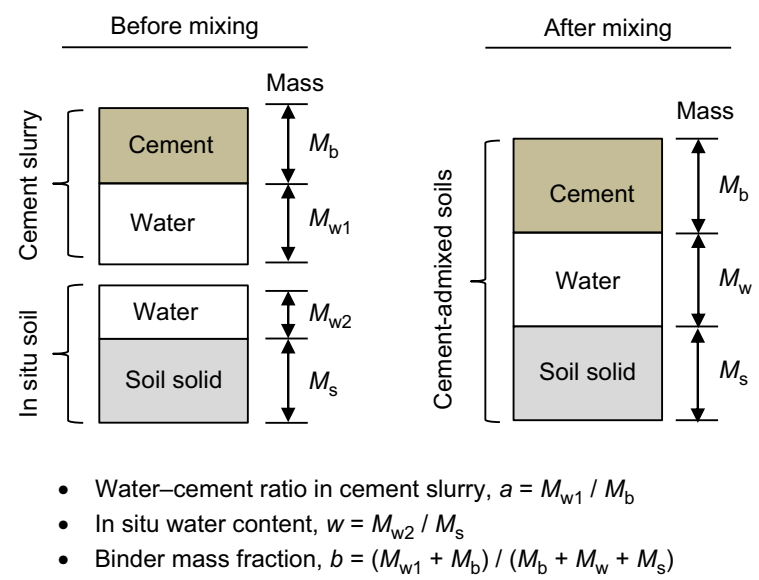

Fig. 6. Sketch of phase relationships before and after mixing of soils

Alternatively, following the approach of Chen et al. (2016), the PDF for the normalised strength can also be derived by expressing the cumulative distribution function (CDF) of the 

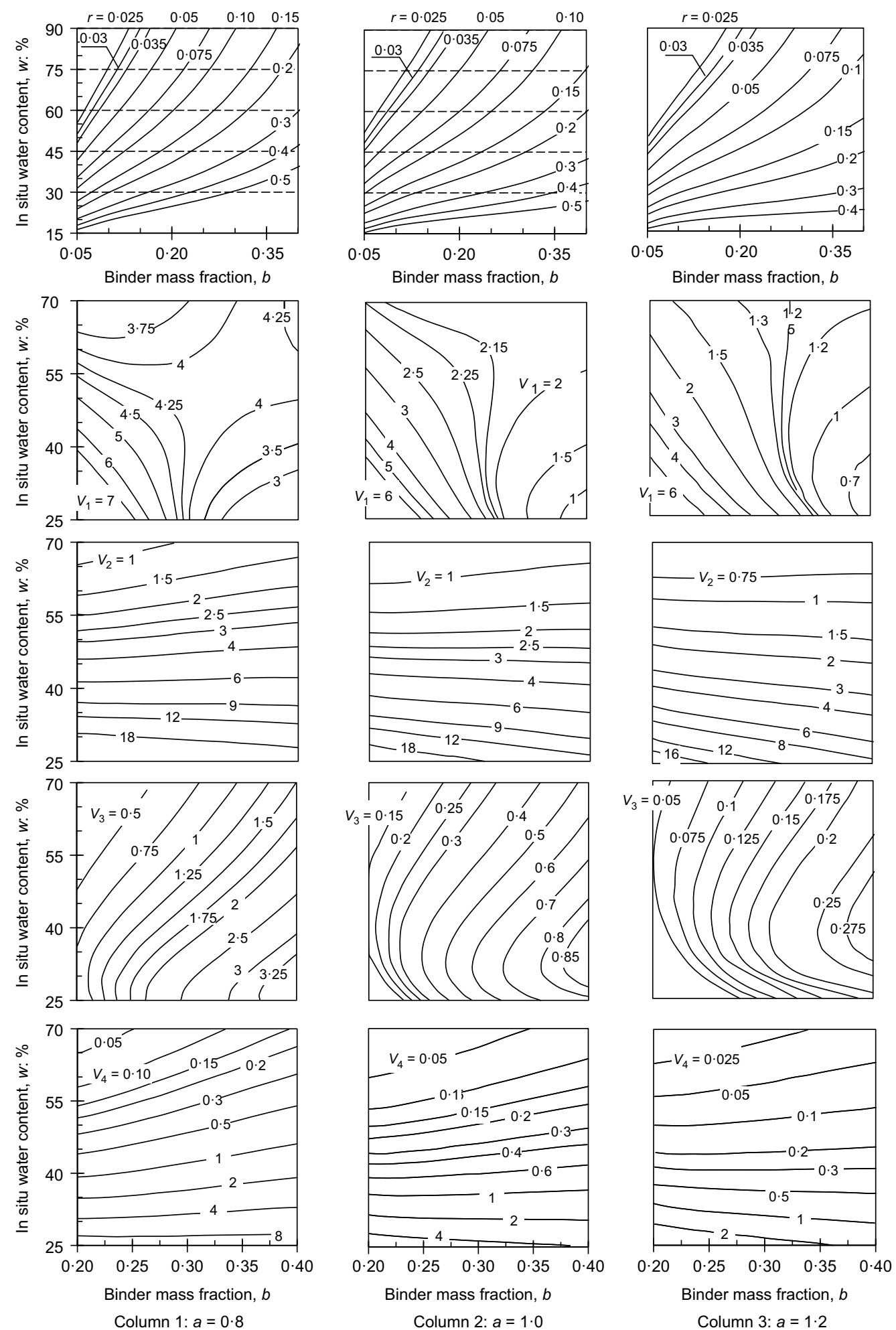

Fig. 7. Tabulated values of $r$ (row 1$), V_{1}$ (row 2$), V_{2}$ (row 3), $V_{3}$ (row 4$)$ and $V_{4}$ (row 5). Rows $2-5$ share the same $x$-axis. $a$ is the water-cement ratio of cement slurry

normalised strength $r, F_{\mathrm{R}}(r)$, as

$$
F_{\mathrm{R}}(r)=P[r(b, w) \leq r]
$$

where $P[-]$ is the probability of an event. Equation (7) can be calculated as

$$
\begin{aligned}
F_{\mathrm{R}}(r) & =\iint_{r(b, w) \leq r} f_{\mathrm{B}, \mathrm{W}}(b, w) \mathrm{d} b \mathrm{~d} w \\
& =\int_{w=0}^{\infty} \int_{b=0}^{r^{-1}} f_{\mathrm{B}, \mathrm{W}}(b, w) \mathrm{d} b \mathrm{~d} w
\end{aligned}
$$

where $f_{\mathrm{BW}}(b, w)$ is the joint PDF of $b$ and $w$, and $r^{-1}=$ $r^{-1}(r, w)$ is the inverse for $b$. Although $b$ and $w$ are assumed to follow the normal distribution (see Table 2), their negative portion (i.e. $-\infty$ to 0 ) is not integrated because of their physical nature. As the variables $b$ and $w$ are independent

$$
f_{\mathrm{B}, \mathrm{W}}(b, w)=f_{\mathrm{B}}(b) f_{\mathrm{W}}(w)
$$

Substituting equation (9) into equation (8) yields

$$
F_{\mathrm{R}}(r)=\int_{w=0}^{\infty} \int_{b=0}^{r^{-1}} f_{\mathrm{B}}(b) f_{\mathrm{W}}(w) \mathrm{d} b \mathrm{~d} w
$$


The PDF of $r, f_{\mathrm{R}}(r)$, can be obtained as

$$
\begin{aligned}
f_{\mathrm{R}}(r) & =\frac{\mathrm{d} F_{\mathrm{R}}(r)}{\mathrm{d} r} \\
& =\int_{w=0}^{\infty}\left|\frac{\partial r^{-1}(r, w)}{\partial r}\right| f_{\mathrm{B}}\left[r^{-1}(r, w)\right] f_{\mathrm{W}}(w) \mathrm{d} w
\end{aligned}
$$

Figure 8 shows the PDF obtained by assuming that the in situ water content and binder distribution are normally distributed with the statistical parameters shown in Table 2. The beta PDF is derived based on the mean and variance from equations (5) and (6), and lower and upper bounds as shown in Table 2. Also plotted in Fig. 8 is a PDF based on equation (11). As the figure shows, the discrepancy between these two distributions is reasonably small. Although equation (11) has a stronger theoretical basis, it is often difficult to evaluate analytically. However, the simplified semi-theoretical method represented by equations (5) and (6) has the advantage that the fitted relationship is explicit, simpler to determine and can be used directly in computeraided numerical simulations.

As a special case where the variance of in situ water content reduces to zero, $f_{\mathrm{W}}(w)$ is only defined at its mean

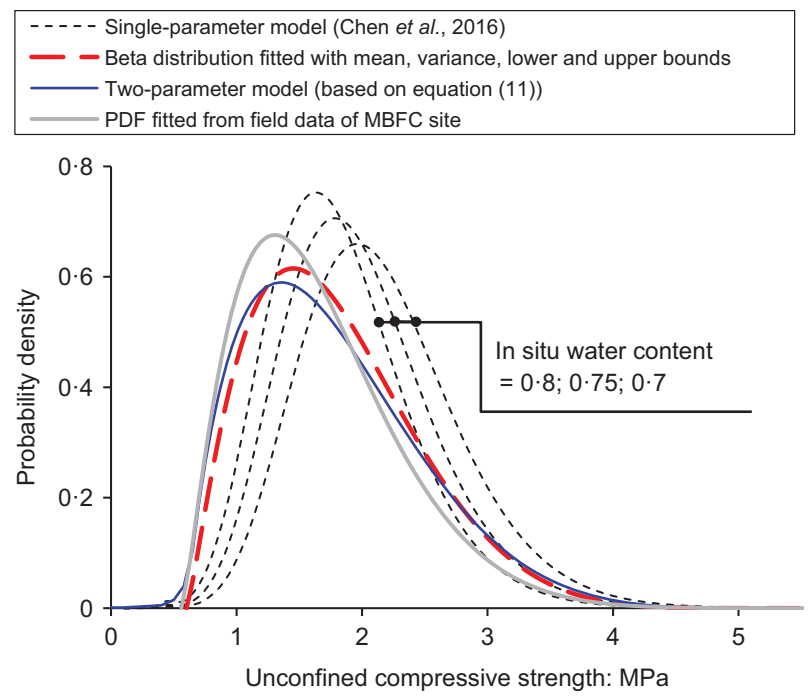

Fig. 8. Comparison among various probability density functions (PDF) predicted from single-parameter and two-parameter models based on the field data set from the MBFC project value $\bar{w}$. Thus, equation (11) reduces to

$$
f_{\mathrm{R}}(r)=\frac{\mathrm{d} F_{\mathrm{R}}(r)}{\mathrm{d} r}=\left|\frac{\mathrm{d} r^{-1}(r, \bar{w})}{\mathrm{d} r}\right| f_{\mathrm{B}}\left[r^{-1}(r, \bar{w})\right]
$$

which is the same as that proposed by Chen et al. (2016). By setting different mean values of in situ water content, equation (12) yields different PDFs, as plotted in Fig. 8 (Chen et al., 2016). As the figure shows, both the fitted beta distribution and the probability distribution computed using equation (11) give a better fit to the MBFC data than the single-parameter model of Chen et al. (2016).

\section{SCALE OF FLUCTUATION}

Chen et al. (2016) did not examine the autocorrelation of the binder concentration in a deep-mixed column. In order to examine the autocorrelation, centrifuge model deep-mixed columns were installed and analysed following the centrifuge modelling procedure of Chen et al. (2016). However, in order to allow samples to be collected at closer equivalent prototype spacings and thereby allow the autocorrelation within a single column to be better measured, larger diameter model columns were installed. Whereas Chen et al.'s tests were conducted with a $50 \mathrm{~mm}$ dia. column under $30 \mathrm{~g}$ model gravity, the current tests were conducted on a $120 \mathrm{~mm}$ dia. column under $20 \mathrm{~g}$ model gravity (see Figs 9 and 10), this being equivalent to $2 \cdot 4 \mathrm{~m}$ dia. columns.

Data were collected from five sections at different depths, as shown in Fig. 9(b), following the sampling locations as shown in Fig. 9(c). The soil samples were taken from the modelled columns by using a miniature scoop, following Lee et al. (2006). An attempt was made to keep the prototype volume size of samples consistent with the size of cored samples from real projects for unconfined compression testing (e.g. $50 \mathrm{~mm}$ dia. $\times 100 \mathrm{~mm}$ high). Following Larsson et al. (2005) and Teo (2017), the autocorrelation was evaluated along the radial and circumferential directions in each section. Along the vertical direction, the autocorrelation was evaluated by considering pairs of data at different depths but with the same radial and circumferential coordinates. Fig. 11 depicts the autocorrelation and $\operatorname{SoF} \theta$ of a model column, which is defined as (Vanmarcke, 1983)

$$
\theta=2 \int_{0}^{\infty} \zeta(\tau) \mathrm{d} \tau
$$

where $\zeta$ is the autocorrelation function and $\tau$ is the distance lag between two points.

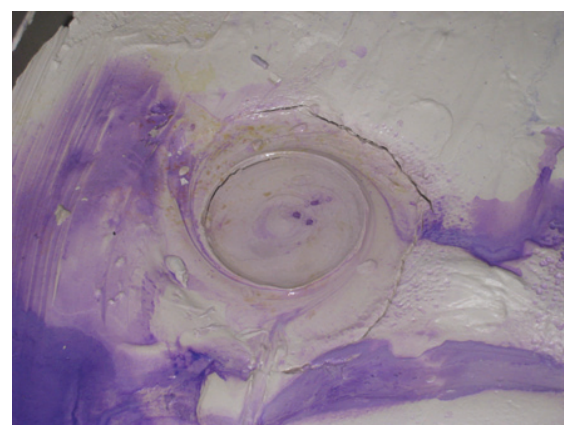

(a)

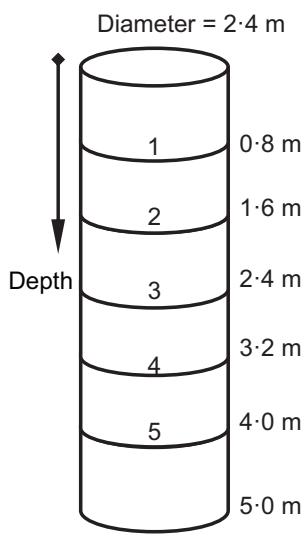

(b)

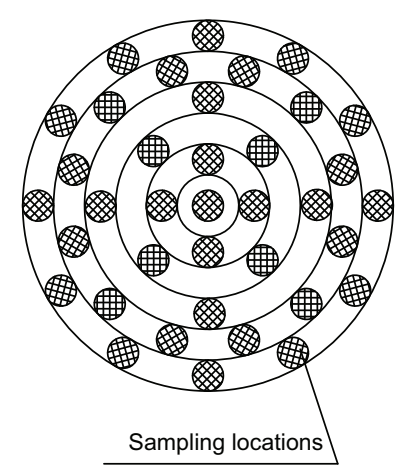

(c)

Fig. 9. (a) Deep-mixing column modelled in centrifuge test. (b) Five sampling depths in a deep-mixing column, where prototype size is shown. (c) Sampling locations in cross-section 


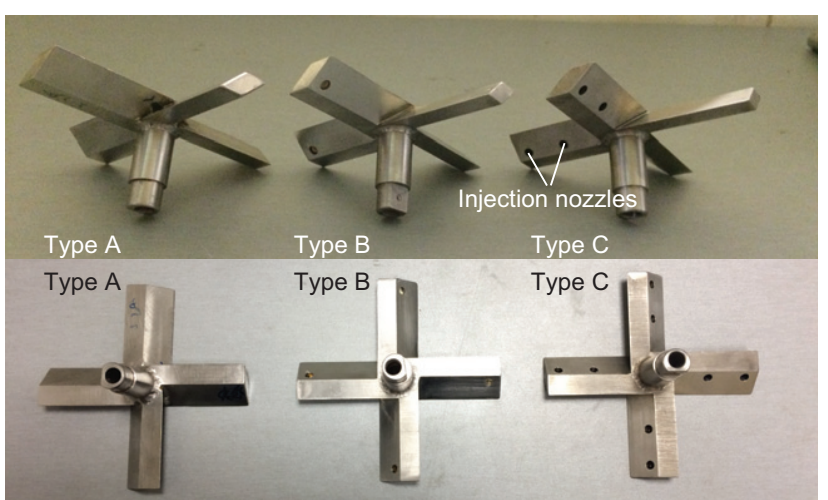

Fig. 10. Deep-mixing installers used in centrifuge modelling tests. Dimensions of installers: width, length and thickness of each wing are $20 \mathrm{~mm}, 100 \mathrm{~mm}$ and $10 \mathrm{~mm}$, respectively; blade angle is $45^{\circ}$. There is only one injection nozzle of the type $A$ installer, located at the installer centre

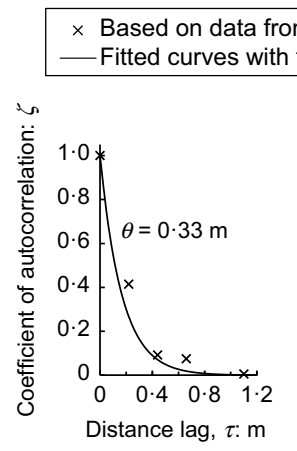

(a)

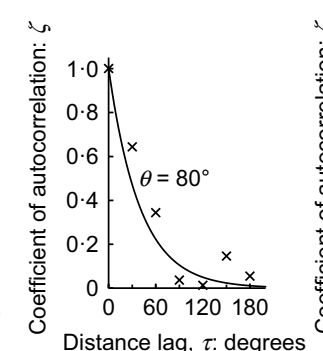

(b)

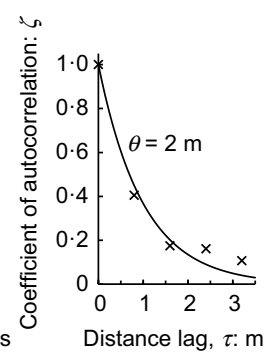

(c)
Fig. 11. Estimation of the SoF, $\theta$, based on data from centrifuge model tests. The values of SoF are calculated based on the fitted curves by way of equation (13): (a) radial direction; (b) circumferential direction; (c) vertical direction

Table 3 summarises the SoF results as well as the operational parameters used in the centrifuge model tests. The centrifuge modelling test procedure and data collection of binder mass fraction were followed as in Chen et al. (2016). The centrifugal results indicate that the OCR has little effect on the SoFs. This may be attributed to the mixing procedure whereby the mixing blades penetrate into the soil first, and then are withdrawn while jetting the binder slurry; the mixing procedure implies that the in situ soils have been disturbed. As also can be seen from Table 3, all of the radial SoFs are significantly smaller than one column diameter, whereas the circumferential SoFs range from one-fifth to one-third of the circumference. Both of these indicate significant variation within a column, which is consistent with the observations of Larsson et al. (2005), but are much smaller than that observed by Navin (2005) and Larsson \& Nilsson (2009). One possible explanation is that the longerrange in situ water content variation (Table 1) may interact with the shorter-range binder concentration. The autocorrelation function of unconfined compressive strength $\zeta$ can be expressed as

$$
\begin{aligned}
\zeta(\tau)= & \int_{-\infty}^{\infty} \int_{-\infty}^{\infty} \frac{\left\{q_{\mathrm{u}}\left(\boldsymbol{u}_{1}\right)-\overline{q_{\mathrm{u}}}\right\}\left\{q_{\mathrm{u}}\left(\boldsymbol{u}_{2}\right)-\overline{q_{\mathrm{u}}}\right\}}{\operatorname{Var}\left[q_{\mathrm{u}}\right]} \\
& \times f\left(\boldsymbol{u}_{1}, \boldsymbol{u}_{2} ; \tau\right) \mathrm{d} \boldsymbol{u}_{1} \mathrm{~d} \boldsymbol{u}_{2}
\end{aligned}
$$

where $\overline{q_{\mathrm{u}}}$ is the mean strength; $\operatorname{Var}[-]$ represents the variance operators; $\boldsymbol{u}_{1}$ and $\boldsymbol{u}_{2}$ are location vectors with the relationship $\left|\boldsymbol{u}_{1}-\boldsymbol{u}_{2}\right|=\tau$; and $f$ is the joint PDF of strength at separation distance $\tau$ and is a function of the PDFs of the in situ water content and binder mass fraction. As the KolmogorovSmirnov test results (Table 2) and the results of Chen et al. (2016) show, the in situ water content and the binder mass fraction can be assumed to follow a normal distribution. Equation (14) is not readily integrated analytically. However, the autocorrelation function can be obtained numerically by the following procedure.

(a) Generate realisations of the random fields of in situ water content and the binder mass fraction using the Monte-Carlo simulations.

(b) Randomly sample the spot in situ water content and the binder mass fraction from the generated random fields.

(c) Calculate the unconfined compressive strength from the sampled in situ water content and the binder mass fraction using equation (1).

Figure 12 shows the estimated coefficient of correlation of strength. As can be seen, convergent and stable estimates of the coefficient of correlation can be obtained using 10000 realisations. Hence, SoFs can be computed by equation (13) numerically from the convergent curves. In this manner, the strength SoF is calculated with results shown in Fig. 13. As Fig. 13(a) shows, the strength SoF depends on the SoF of the binder concentration and that of the in situ water content. Moreover, if the in situ water content is uniform, the strength SoF is almost equal to that of the binder concentration, Fig. 13(b), as expected. The results in Table 1 indicate that the SoF of binder concentration $b$ is often intra-column level, whereas the SoF of in situ water content $w$ is inter-column level. As a result, two levels of strength SoF can be derived based on the region size of the problem under consideration. This can be examined by numerically simulating a cementtreated slab, and then sampling within various region sizes of the slab.

Figures 14(a)-14(c) illustrate the variation in the in situ water content, binder concentration and resulting strength within a simulated region comprising around 700 columns. The random fields of in situ water content and binder concentration were generated by the modified linear estimation method (see Liu et al., 2014), whereby the resultant strength was calculated with equation (1). As Table 1 and Fig. 14(a) illustrate, variation of the in situ water content of a ground typically has an SoF that is much greater than the diameter of a deep-mixing column and therefore much greater than that of binder variation illustrated in Fig. 14(b). As Fig. 14(c) shows, at longer range, the strength distribution reflects the variation in the in situ water content, which spans across several columns. However, there is also a short-range variation, which reflects the variation in binder concentration.

Figure 15(a) shows the change in the horizontal SoF, estimated from spot sampling of strength distribution within a square-shaped region with dimensions $s \times s$, as the dimension $s$ varies. The strength values were collected by randomly taking point values from the simulated strength field (Fig. 14(c)) within the prescribed sample region. When the size of the sample region is small, the horizontal strength SoF approaches that of binder concentration (e.g. Larsson et al., 2005). This reflects the case in which sampling is conducted column by column. As the size $s$ increases, the horizontal strength SoF increases to reflect that of the in situ water content. This is consistent with the data of Navin (2005), which show the horizontal SoF that is much greater than the column diameter. Thus, the results of SoF in unconfined compressive strength depend on the size of the sampled region. However, the vertical SoF remains largely unchanged at approximately $2 \cdot 8 \mathrm{~m}$, Fig. 15(b). This is 
Table 3. Results of centrifuge modelling tests

\begin{tabular}{|c|c|c|c|c|c|c|c|c|c|c|}
\hline \multirow{2}{*}{$\begin{array}{l}\text { Column } \\
\text { ID }\end{array}$} & \multirow{2}{*}{$\begin{array}{l}\text { Overconsolidation } \\
\text { ratio }\end{array}$} & \multirow{2}{*}{$\begin{array}{c}\text { Prototype } \\
\text { rotational speed: } \\
\mathrm{r} / \mathrm{min}\end{array}$} & \multirow{2}{*}{$\begin{array}{l}\text { Binder } \\
\text { density: } \\
\mathrm{g} / \mathrm{cm}^{3}\end{array}$} & \multirow{2}{*}{$\begin{array}{l}\text { Withdrawal } \\
\text { rate: } \mathrm{m} / \mathrm{min}\end{array}$} & \multirow{2}{*}{$\begin{array}{l}\text { Mixing } \\
\text { installer } \\
\text { type* }\end{array}$} & \multirow{2}{*}{$\begin{array}{l}\text { Blade } \\
\text { rotational } \\
\text { number }\end{array}$} & \multirow{2}{*}{$\begin{array}{c}\text { Centrifuge } \\
g \text {-level }\end{array}$} & \multicolumn{3}{|c|}{ Scale of fluctuation (SoF) } \\
\hline & & & & & & & & $\begin{array}{l}\text { Vertical: } \\
\text { m }\end{array}$ & $\begin{array}{c}\text { Radial } \\
\text { SoF/diameter } \dagger\end{array}$ & $\begin{array}{c}\text { Circumferential: } \\
\text { degrees }\end{array}$ \\
\hline DM1 & 1 & 18 & 1.5 & $0 \cdot 2$ & A & 360 & 20 & $2 \cdot 00$ & $0 \cdot 14$ & 80 \\
\hline DM2 & 1 & 8 & $1 \cdot 5$ & 0.09 & A & 360 & 20 & $2 \cdot 00$ & $0 \cdot 18$ & 100 \\
\hline DM3 & 1 & 10 & $1 \cdot 5$ & $0 \cdot 11$ & A & 360 & 20 & $2 \cdot 22$ & $0 \cdot 14$ & 80 \\
\hline DM4 & 1 & 12 & $1 \cdot 5$ & $0 \cdot 13$ & A & 360 & 20 & $3 \cdot 33$ & $0 \cdot 21$ & 80 \\
\hline DM5 & 1 & 14 & 1.5 & $0 \cdot 15$ & A & 360 & 20 & $2 \cdot 00$ & $0 \cdot 17$ & 80 \\
\hline DM6 & 1 & 16 & $1 \cdot 5$ & $0 \cdot 18$ & A & 360 & 20 & $2 \cdot 00$ & $0 \cdot 13$ & 100 \\
\hline DM7 & 4 & 30 & $1 \cdot 5$ & $0 \cdot 33$ & A & 360 & 20 & $2 \cdot 00$ & $0 \cdot 17$ & 100 \\
\hline DM8 & 4 & 18 & 1.5 & $0 \cdot 2$ & A & 360 & 20 & $3 \cdot 33$ & $0 \cdot 21$ & 100 \\
\hline DM9 & 4 & 10 & $1 \cdot 5$ & $0 \cdot 11$ & A & 360 & 20 & $2 \cdot 67$ & $0 \cdot 13$ & 100 \\
\hline DM10 & 4 & 12 & $1 \cdot 5$ & $0 \cdot 13$ & A & 360 & 20 & $3 \cdot 33$ & $0 \cdot 15$ & 100 \\
\hline DM11 & 4 & 14 & 1.5 & $0 \cdot 15$ & A & 360 & 20 & $2 \cdot 00$ & $0 \cdot 17$ & 67 \\
\hline DM12 & 4 & 16 & $1 \cdot 5$ & $0 \cdot 18$ & A & 360 & 20 & $2 \cdot 00$ & $0 \cdot 24$ & 100 \\
\hline DM13 & 1 & 10 & $1 \cdot 5$ & $0 \cdot 11$ & B & 360 & 20 & $2 \cdot 86$ & $0 \cdot 18$ & 87 \\
\hline DM14 & 1 & 12 & 1.5 & $0 \cdot 13$ & B & 360 & 20 & $2 \cdot 67$ & $0 \cdot 21$ & 100 \\
\hline DM15 & 1 & 14 & 1.5 & $0 \cdot 15$ & B & 360 & 20 & 3.00 & $0 \cdot 13$ & 67 \\
\hline DM16 & 1 & 16 & $1 \cdot 5$ & $0 \cdot 18$ & $\mathrm{~B}$ & 360 & 20 & $2 \cdot 00$ & $0 \cdot 21$ & 100 \\
\hline DM17 & 1 & 18 & $1 \cdot 5$ & $0 \cdot 2$ & $\mathrm{~B}$ & 360 & 20 & $2 \cdot 00$ & $0 \cdot 24$ & 67 \\
\hline DM18 & 1 & 30 & 1.5 & 0.33 & B & 360 & 20 & $2 \cdot 00$ & $0 \cdot 12$ & 80 \\
\hline DM19 & 1 & 10 & $1 \cdot 5$ & $0 \cdot 11$ & $\mathrm{C}$ & 360 & 20 & $2 \cdot 50$ & $0 \cdot 21$ & 67 \\
\hline DM20 & 1 & 12 & $1 \cdot 5$ & $0 \cdot 13$ & $\mathrm{C}$ & 360 & 20 & $2 \cdot 00$ & $0 \cdot 14$ & 67 \\
\hline DM21 & 1 & 14 & 1.5 & $0 \cdot 15$ & $\mathrm{C}$ & 360 & 20 & $2 \cdot 22$ & $0 \cdot 14$ & 87 \\
\hline DM22 & 1 & 16 & $1 \cdot 5$ & $0 \cdot 18$ & $\mathrm{C}$ & 360 & 20 & 1.33 & $0 \cdot 17$ & 87 \\
\hline DM23 & 1 & 18 & $1 \cdot 5$ & $0 \cdot 2$ & $\mathrm{C}$ & 360 & 20 & $1 \cdot 00$ & $0 \cdot 24$ & 100 \\
\hline DM24 & 1 & 30 & $1 \cdot 5$ & $0 \cdot 33$ & $\mathrm{C}$ & 360 & 20 & $2 \cdot 00$ & $0 \cdot 13$ & 100 \\
\hline DM25 & 4 & 10 & $1 \cdot 5$ & $0 \cdot 11$ & $\mathrm{~B}$ & 360 & 20 & $3 \cdot 33$ & $0 \cdot 18$ & 87 \\
\hline DM26 & 4 & 12 & $1 \cdot 5$ & $0 \cdot 13$ & $\mathrm{~B}$ & 360 & 20 & $2 \cdot 67$ & $0 \cdot 21$ & 80 \\
\hline $\mathrm{DM} 27$ & 4 & 14 & $1 \cdot 5$ & $0 \cdot 15$ & $\mathrm{~B}$ & 360 & 20 & $1 \cdot 00$ & $0 \cdot 14$ & 100 \\
\hline DM28 & 4 & 16 & $1 \cdot 5$ & $0 \cdot 18$ & B & 360 & 20 & $2 \cdot 00$ & $0 \cdot 15$ & 67 \\
\hline DM29 & 4 & 18 & $1 \cdot 5$ & $0 \cdot 2$ & B & 360 & 20 & $1 \cdot 33$ & $0 \cdot 17$ & 80 \\
\hline DM30 & 4 & 30 & 1.5 & $0 \cdot 33$ & $\mathrm{~B}$ & 360 & 20 & 2.67 & $0 \cdot 14$ & 133 \\
\hline DM31 & 4 & 10 & $1 \cdot 5$ & $0 \cdot 11$ & $\mathrm{C}$ & 360 & 20 & $1 \cdot 00$ & $0 \cdot 28$ & 67 \\
\hline DM32 & 4 & 12 & $1 \cdot 5$ & $0 \cdot 13$ & $\mathrm{C}$ & 360 & 20 & $2 \cdot 00$ & $0 \cdot 21$ & 87 \\
\hline DM33 & 4 & 14 & $1 \cdot 5$ & $0 \cdot 15$ & $\mathrm{C}$ & 360 & 20 & $2 \cdot 00$ & $0 \cdot 18$ & 87 \\
\hline DM34 & 4 & 16 & 1.5 & $0 \cdot 18$ & $\mathrm{C}$ & 360 & 20 & $1 \cdot 33$ & $0 \cdot 21$ & 100 \\
\hline DM35 & 4 & 18 & $1 \cdot 5$ & $0 \cdot 2$ & $\mathrm{C}$ & 360 & 20 & $2 \cdot 00$ & $0 \cdot 17$ & 80 \\
\hline DM36 & 4 & 30 & $1 \cdot 5$ & $0 \cdot 33$ & $\mathrm{C}$ & 360 & 20 & $2 \cdot 67$ & $0 \cdot 14$ & 133 \\
\hline \multicolumn{8}{|c|}{ Minimum of SoF: } & $1 \cdot 0$ & $0 \cdot 12$ & 67 \\
\hline \multicolumn{8}{|c|}{ Average of SoF: } & $2 \cdot 18$ & $0 \cdot 17$ & 89 \\
\hline \multicolumn{8}{|c|}{ Maximum of SoF: } & $3 \cdot 33$ & $0 \cdot 28$ & 133 \\
\hline \multicolumn{8}{|c|}{ Coefficient of variation of SoF: } & $0 \cdot 3$ & $0 \cdot 23$ & $0 \cdot 19$ \\
\hline
\end{tabular}

* Mixing installer types A, B and C are illustrated in Fig. 10.

$\dagger$ Column diameter in prototype is $2.4 \mathrm{~m}$. 


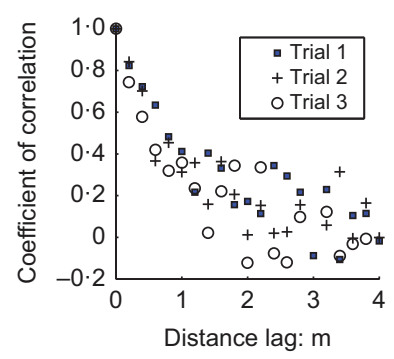

(a)

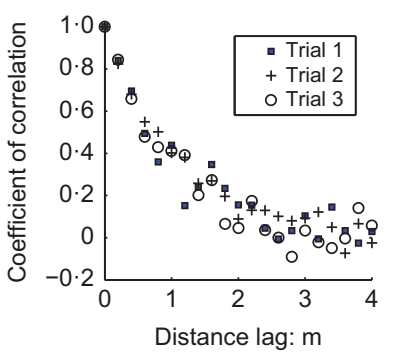

(b)

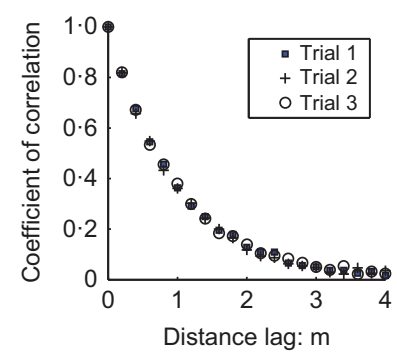

(c)

Fig. 12. Estimation of autocorrelation function of strength based on $N$ realisations of binder concentration random field $(\mathrm{SoF}=0 \cdot 5 \mathrm{~m})$ and in situ water content random field $(\mathrm{SoF}=6 \mathrm{~m}):(\mathrm{a}) N=50$; (b) $N=100$; (c) $N=10000$

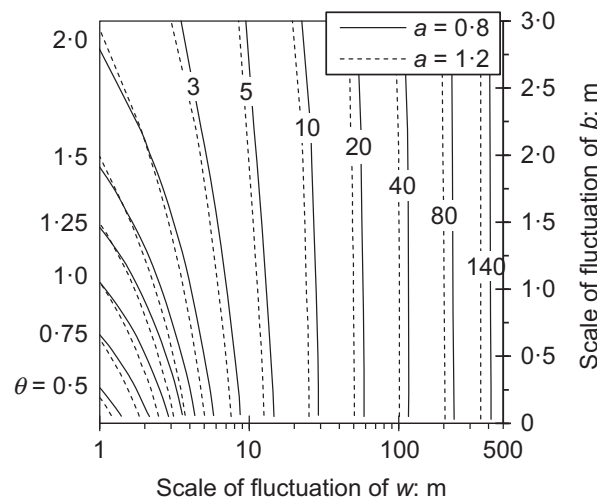

(a)

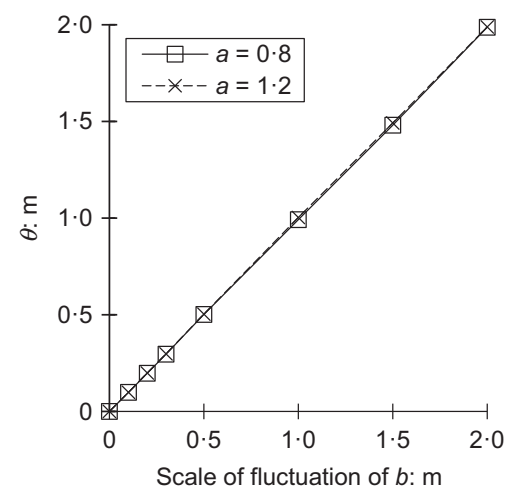

(b)

Fig. 13. Relationship among scales of fluctuation of unconfined compressive strength, binder mass fraction $b$ and in situ water content $w$ for cement-slurry water-cement mass ratio $a=0 \cdot 8$ and 1.2: (a) two-parameter prediction model; (b) single-parameter prediction model with in situ water content being assumed to be constant. $\theta=$ strength SoF

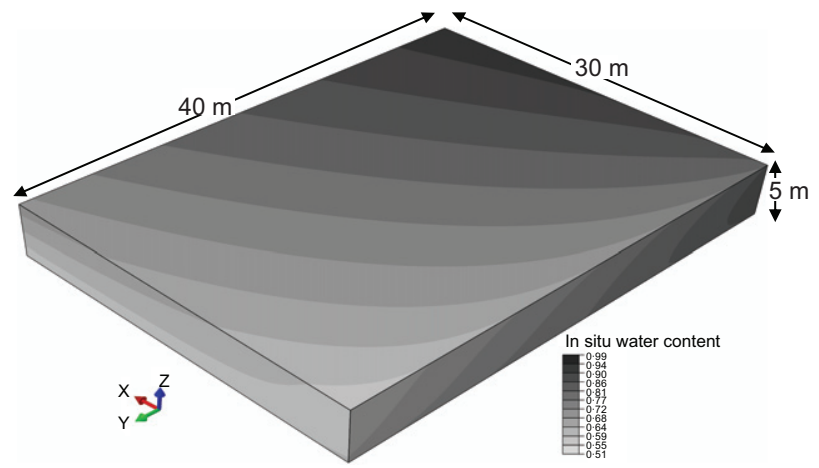

(a)

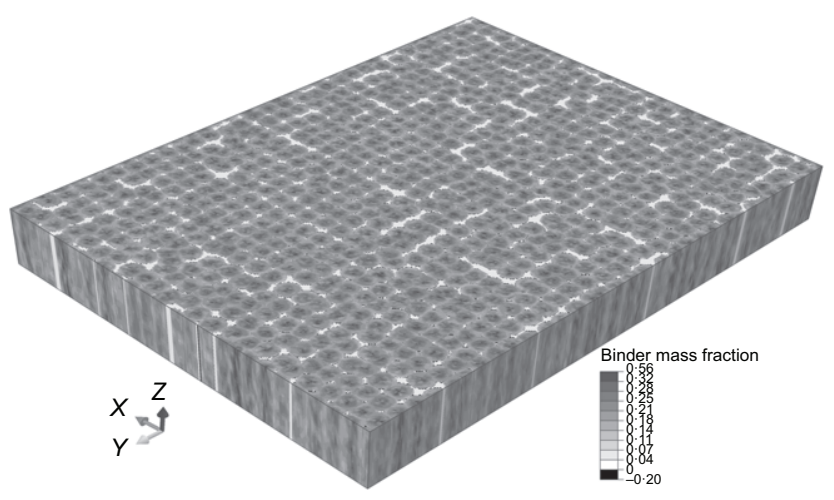

(b)

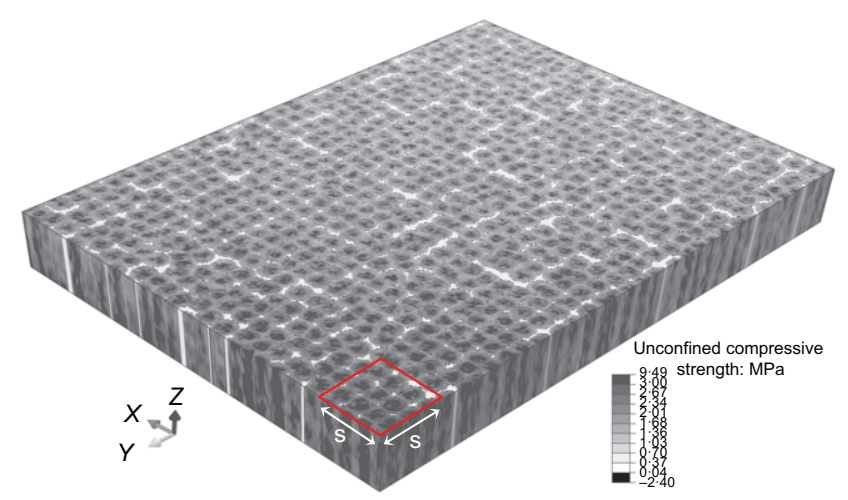

(c)

Fig. 14. Illustration of the effect of in situ water content on the unconfined compressive strength: (a) a realisation of in situ water content with horizontal and vertical scales of fluctuation of $70 \mathrm{~m}$ and $6 \mathrm{~m}$, respectively; (b) a realisation of binder mass fraction with the (average) statistical parameters listed in Table 1 and a positioning error of columns of $0.35 \mathrm{~m}$; (c) contour of resulting unconfined compressive strength. The small portions with negative values in (b) and (c) are introduced by the software in extrapolating data. The determination and simulation of the positioning error can be found in Liu et al. (2015) 


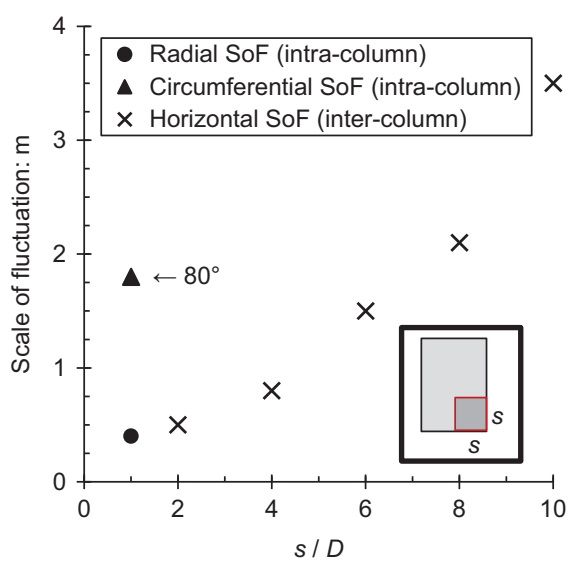

(a)

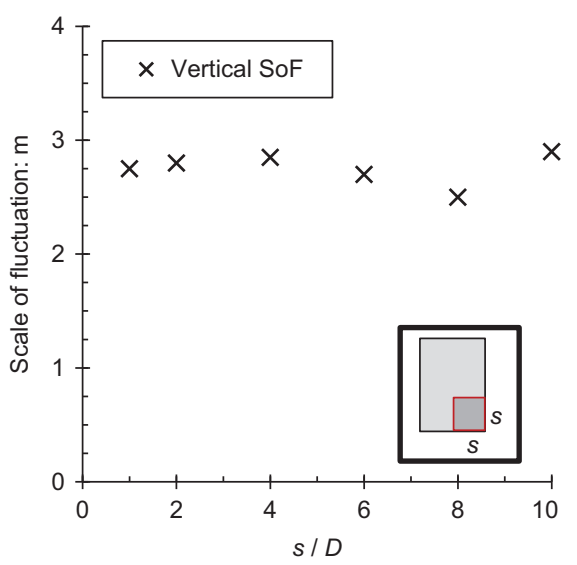

(b)

Fig. 15. (a) Horizontal; (b) vertical scales of fluctuation (SoF) based on samples from the numerical simulation in Fig. 14(c). $(D=$ column diameter; $s=$ size of sampled region)

Table 4. Case study of statistical prediction model for UCS of cement-admixed clays

\begin{tabular}{|c|c|c|c|c|c|c|}
\hline \multicolumn{3}{|c|}{ Statistical characteristics } & \multirow{3}{*}{$\begin{array}{l}\text { Binder mass } \\
\text { fraction, } b \\
0 \cdot 28 \\
0.10\end{array}$} & \multirow{3}{*}{$\begin{array}{l}\text { In situ water content, } \\
w \\
0.69\end{array}$} & $\begin{array}{l}\text { Predicted } \\
\text { UCS } \\
\left(q_{\mathrm{u}}=r \times q_{0} *\right) \\
\end{array}$ & $\begin{array}{l}\text { Measured } \\
\text { UCS, } q_{\mathrm{u}}\end{array}$ \\
\hline Probability & MBFC site & Mean & & & $1.9 \mathrm{MPa}$ & $1.7 \mathrm{MPa}$ \\
\hline distribution & $(a=0.9)$ & $\mathrm{CoV}$ & & & $0 \cdot 35$ & $0 \cdot 42$ \\
\hline related statistics & & Standard deviation & $0 \cdot 053$ & $0 \cdot 055$ & $0.67 \mathrm{MPa}$ & $0.71 \mathrm{MPa}$ \\
\hline & & Lower bound & $0 \cdot 12$ & $0 \cdot 53$ & $0 \cdot 5 \mathrm{MPa}$ & $0.62 \mathrm{MPa}$ \\
\hline & & Upper bound & $0 \cdot 44$ & $0 \cdot 78$ & $5 \cdot 6 \mathrm{MPa}$ & $5 \cdot 34 \mathrm{MPa}$ \\
\hline & Marina One & Mean & $0 \cdot 19$ & $0 \cdot 47$ & $2 \cdot 2 \mathrm{MPa}$ & $2 \cdot 1 \mathrm{MPa}$ \\
\hline & site $(a=1 \cdot 0)$ & $\mathrm{CoV}$ & $0 \cdot 29$ & $0 \cdot 16$ & $0 \cdot 39$ & $0 \cdot 44$ \\
\hline & & Standard deviation & $0 \cdot 055$ & $0 \cdot 075$ & $0 \cdot 86 \mathrm{MPa}$ & $0.93 \mathrm{MPa}$ \\
\hline & & Lower bound & $0 \cdot 03$ & $0 \cdot 20$ & $0 \cdot 32 \mathrm{MPa}$ & $0.68 \mathrm{MPa}$ \\
\hline & & Upper bound & $0 \cdot 35$ & $0 \cdot 66$ & $11 \cdot 2 \mathrm{MPa}$ & 7·0 MPa \\
\hline \multirow[t]{4}{*}{ Scale of fluctuation } & Vertical & & $1-3 \cdot 33 \mathrm{~m}$ & 6 m (see Fig. 4(c)) & $2 \cdot 6-4 \cdot 3 \mathrm{~m}$ & $\begin{array}{l}3 \cdot 3 \mathrm{~m} \text { (see } \\
\text { Fig. 5(a)) }\end{array}$ \\
\hline & Horizontal & Inter-column & $0 \cdot 12-0 \cdot 28 D^{\dagger}$ & $\begin{array}{l}\text { 100-200 m } \\
\text { (Singapore marine } \\
\text { clay, Table 1) }\end{array}$ & $35-40 \mathrm{~m} t$ & $\begin{array}{l}25 \mathrm{~m}(\mathrm{see} \\
\text { Fig. 5(b)) }\end{array}$ \\
\hline & & $\begin{array}{l}\text { Radial } \\
\quad \text { (intra-column) }\end{array}$ & $0 \cdot 12-0 \cdot 28 D$ & Constant & $0 \cdot 12-0 \cdot 28 D$ & - \\
\hline & & $\begin{array}{l}\text { Circumferential } \\
\text { (intra-column) }\end{array}$ & $67-133^{\circ}$ & Constant & $67-133^{\circ}$ & - \\
\hline
\end{tabular}

$* q_{0}=20 \mathrm{MPa}$ for 28 day curing time (see Xiao et al., 2014).

$\dagger D=$ column diameter, which is $2.4 \mathrm{~m}$ (prototype) in centrifuge model in this study.

$\$$ This range is applicable for Marina One project, where the SoF of in situ water content is $100 \mathrm{~m}$, as shown in Fig. 4(b).

because sampling is conducted over the entire column length, which is independent of the size of the sampled region $s$.

\section{COMPARISON WITH FIELD DATA}

Table 4 shows the measured statistical parameters and those predicted using the framework presented above. The lower and upper bounds of binder mass fraction and in situ water content were taken from centrifuge model results and field data, respectively. In the Marina One site, the marine clay was divided into two layers designated $2 \mathrm{~A}$ and $2 \mathrm{~B}$, as shown in Fig. 2. Approximately $58 \%$ of the available in situ water content data are from layer $2 \mathrm{~A}$ and $42 \%$ from layer $2 \mathrm{~B}$. This fraction is proportional to the volume fraction between these two layers, as the boreholes are generally uniformly distributed in the site. As Table 4 and Fig. 16(a) show, the computed strength of the cement-treated ground in these two layers has significantly different statistics and PDFs. The mean strength and strength $\mathrm{CoV}$ of layer $2 \mathrm{~B}$ are much higher than the corresponding parameters of layer $2 \mathrm{~A}$, owing to the lower mean in situ water content and higher in situ water content CoV. The total number of core strength data points from the Marina One site is 1145 (Liu et al., 2017). However, the measured core strength data of the two layers of treated soil were not separately classified, and it was not possible to analyse the core strength statistics separately in two layers. Hence, as shown in Table 4, only one set of statistical parameters for strength was reported; these lie in between those of the treated layers $2 \mathrm{~A}$ and $2 \mathrm{~B}$.

To assess whether the computed statistical parameters for layers $2 \mathrm{~A}$ and $2 \mathrm{~B}$ are consistent with those of measured core strength data, a method of combining the two computed distributions in Fig. 16(a) is devised. First, the proportion of in situ water content data is assumed to be equal to the volume ratio of the two layers of marine clay. Second, it is assumed that this volume ratio is also reflected in the core strength data; that is, $58 \%$ of the core strength data come from layer $2 \mathrm{~A}$ and $42 \%$ from layer $2 \mathrm{~B}$. Based on this, a set of 1145 core strength data points were then generated using the 
distributions in Fig. 16(a), with $58 \%$ of the data points from layer $2 \mathrm{~A}$ and $42 \%$ from layer $2 \mathrm{~B}$. As Fig. 16(b) shows, the histogram of the simulated core strength data agrees reasonably well with that of the measured strength data. Moreover, as Table 4 shows, the mean and CoV of the simulated combined data agree well with the measured values. The CoVs computed based on equations (5) and (6) are slightly lower than the measured values. This is in part because the unconfined compression tests themselves are likely to introduce additional random testing error that increases the variation. Extensive discussion on the magnitude of random testing error can be found in Baecher \& Christian (2003). As mentioned earlier, the computed PDFs using equations (5) and (6) with a beta distribution as well as equation (12) give a better fit to the measured distribution than that of Chen et al. (2016), Fig. 8.

Table 4 also compares the strength SoF derived from Fig. 13. As can be seen, the computed vertical SoF is in reasonable agreement with the measured value from the MBFC and Marina One sites. In particular, neither of the two sites yielded any intra-column data, as the lateral core spacing typically spanned over several column diameters. Direct comparison cannot be made with previous studies as the specific processes and operational parameters of the different studies are dissimilar. However, the computed inter-column SoF is consistent with and would explain the large horizontal value reported by Navin (2005), Table 1. The intra-column radial SoF also agrees reasonably well with that of Larsson et al. (2005), although the process used is different. This suggests that the intracolumn SoF is reasonably well reflected by centrifuge modelling tests. In the vertical direction, the computed strength SoF is also quite close to that deduced from the core strength data.

\section{ENGINEERING IMPLICATIONS}

The effect of dimension of the sampled region on the measured random and spatial variation of the strength of cement-treated ground has still not been widely studied to date. What has been documented in the literature is the significant divergence in the measured values of $\mathrm{CoV}$ and SoF. However, this phenomenon has not been satisfactorily explained and quantified to date. The main contribution herein is not merely the documentation of this phenomenon, but also its explanation in terms of the interaction between in situ water content and the quality of mixing effect and the quantification of this interaction, both in terms of the $\mathrm{CoV}$ and SoF.

In terms of $\mathrm{CoV}$, the interaction is reflected in equation (6). This represents a refinement of the model by Chen et al. (2016) for predicting variation of strength in treated ground. Chen et al. (2016) proposed a statistical model for predicting the spatial variation of strength in cement-treated ground based on the density of the cement slurry and the blade rotation number. In the model by Chen et al. (2016), the in situ water content was assumed to be uniform and possible variation in the in situ water content was examined using parametric studies. By contrast, the present study considered the variation of the in situ water content in equation (6). Hence, from the viewpoint of the design of cement treatment schemes, equation (6) provides a method of estimating the overall variance in strength if the variance in binder mass fraction can be estimated by way of the density of the cement slurry and blade rotation number and the variance of the in situ water content can be estimated from site investigation data.

Apart from design, the findings of this study also have implications for the quality control of deep mixing operations. Equation (6) indicates that the measured variation

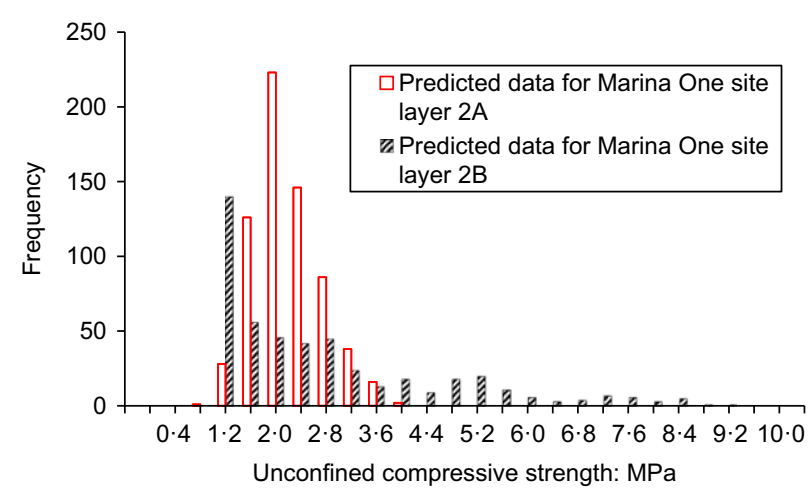

(a)

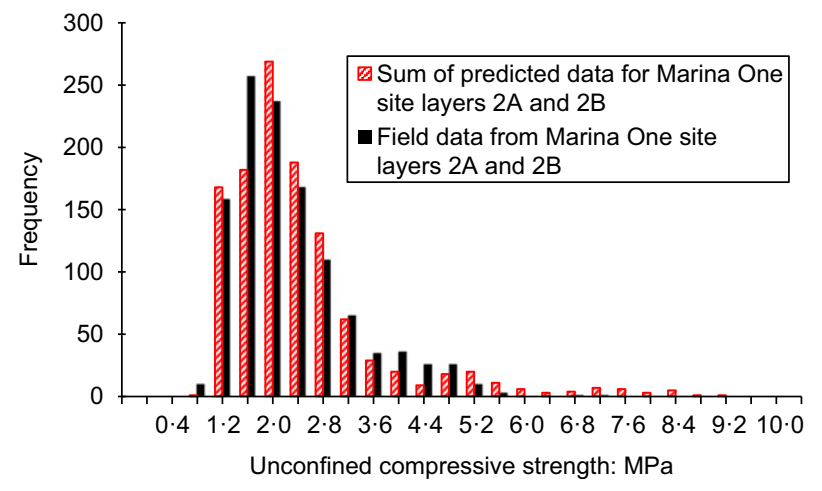

(b)

Fig. 16. (a) Predicted data for two layers in Marina One site. (b) Comparison between histograms from predicted and field data (layer 2A: upper marine clay; layer 2B: lower marine clay)

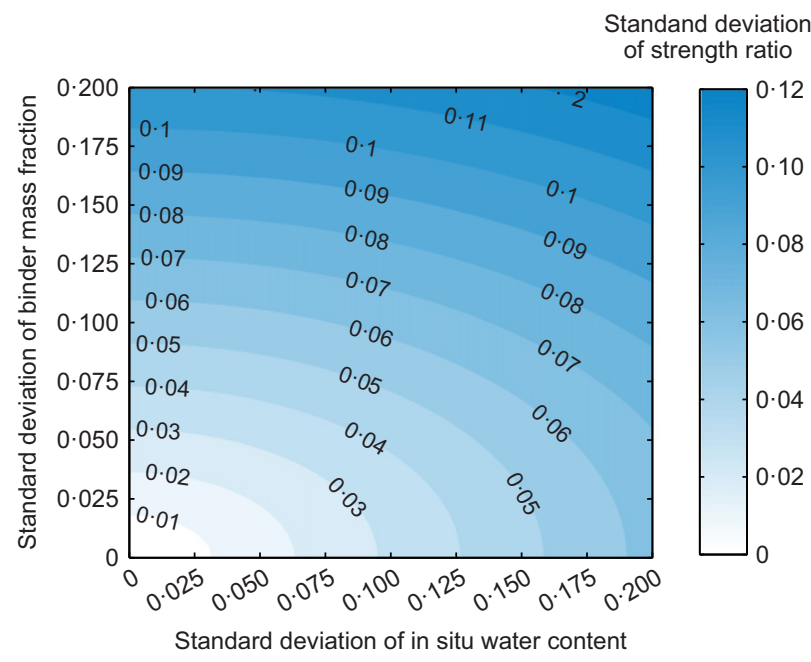

Fig. 17. Relationship between standard deviations among strength ratio $(r)$, in situ water content $(w)$ and binder mass fraction $(b)$. The mean values of $w$ and $b$ are based on the MBFC project

in strength ratio $(r)$ consists of two components from the variation of in situ water content over the area of measurement $(w)$ and binder mass fraction $(b) . V_{3}$ and $V_{4}$ depend on the mean values of $b$ and $w$, and can be determined using the relationships in Appendix 1. As a result, one of the variances can be calculated as long as the other two variances (or standard deviations) are given, as illustrated in Fig. 17. In an actual deep-mixing project, the measured variance is not a function of the mixing quality alone. From the perspective of quality control, a better indicator of the mixing quality is $\operatorname{Var}[b]$. Equations (6a) and (6b) allow $\operatorname{Var}[b]$ or 
$\mathrm{CoV}[b]$ to be estimated if the variance of the in situ water content $\operatorname{Var}[w]$ is known from site investigation data.

In terms of SoF, the results highlight the co-existence of SoF in the intra- or inter-column levels. In large cement treatment projects, the dimension of the treatment area is likely to be of a similar order or greater than the SoF of in situ water content. Moreover, the SoF in the in situ water content is typically much greater than that of the binder concentration. The presence of two very different SoFs has implications for core sampling, quality control and simulation of cement-treated soil. The long-range SoF may be important for overall performance of a cement-treated soil mass, whereas the short-range SoF has a significant influence on the strength and stiffness of individual column (e.g. Namikawa \& Koseki, 2013). In core sampling for quality control of cement deep-mixing works, the spacing between boreholes is typically much greater than a column diameter. For instance, in Marina One, the typical borehole spacing is about $10 \mathrm{~m}$. At such spacings, the mean and $\mathrm{CoV}$ of the spatial distribution may still be reflected, but the short-range intra-column SoF, which is more reflective of the mixing quality, will not. Hence, at least some of the boreholes should be conducted at close spacing of less than a column diameter if intra-column variation is to be captured. Conversely, in simulating random fields for cement-treated ground, an SoF that is much greater than a column diameter would imply that the strength within each column is quite uniform, which may not be realistic.

\section{CONCLUDING REMARKS}

The foregoing discussion shows that, apart from variation in binder concentration, the variation of in situ water content also has a significant effect on the variation in strength of the cement-mixed ground. By considering the spatial variation of binder concentration and in situ water content together within the theoretical framework developed above, improved estimates of the mean strength and strength $\mathrm{CoV}$ are obtained compared with that derived by just considering the variation in binder concentration (e.g. Chen et al., 2016).

The interaction between variations in binder concentration and in situ water content can give rise to two levels of variation. Variation in binder concentration occurs at an intracolumn level and gives rise to a short-range horizontal SoF that can be manifest by restricting the size of the sampling region to about one column diameter. This short-range strength SoF is approximately equal to that of the variation in binder concentration within a column. At such short range, variation of the in situ water content is insignificant. This short-range SoF can be reflected in centrifuge model data.

The SoF of in situ water content variation is typically much greater than a column diameter. Its interaction with the variation in binder concentration is to give rise to a long-range inter-column strength $\mathrm{SoF}$ that is much greater than a column diameter as well as the short-range SoF due to binder concentration variation alone. The presence of short- and long-range SoFs provides an explanation for the wide range of measured strength $\mathrm{SoF}$ in cement-mixed ground. Both these SoFs can be determined using the framework proposed above. The presence of two very different SoFs has implications on core sampling, quality control and simulation of cement-treated soil. The long-range SoF may be important for overall performance of a cement-treated soil mass, whereas the short-range SoF has a significant influence on the strength and stiffness of individual column. In core sampling for quality control of cement deep-mixing works, the spacing between boreholes is typically much greater than a column diameter. At least some of the boreholes should be conducted at close spacings of less than a column diameter if intra-column variation is to be captured.

The relationship among the variances of strength ratio, in situ water content and binder mass fraction is established (i.e. equation (6)). With this relationship, field mixing quality can be estimated as long as the variances of the in situ water content and binder mass fraction are known. However, according to the relationship of equation (6), if the requirement of overall uniformity of a deep-mixing project is pre-determined, the required variance of binder mass fraction can be achieved by altering the blade rotation number accordingly. The crux of this study is the method of deriving the variation in mix ratios and deducing from it the strength of the resultant mix using equation (1). If another alternative relationship is shown to be more appropriate for another soil type, one can still readily apply equations (5) and (6) using a different relationship between the mix ratios (i.e. $x$ and $y$ ) and $r$. Some transformation error (see Zhang et al. (2018)) may be induced by equation (1) or any alternative correlation between mix ratios and strength. This transformation error was not taken into account in the current study and will be the subject of future studies.

\section{ACKNOWLEDGEMENTS}

This research is supported by National Natural Science Foundation of China (51608218), the National Research Foundation Singapore under its Competitive Research Programme (CRP award no. NRF-CRP 6-2010-03) and the R\&D project (2016-SK-1) from Shanghai Tunnel Engineering Corporation. The second and third authors would also like to acknowledge the research scholarship support provided by the National University of Singapore.

\section{APPENDIX 1. DERIVATIONS OF COEFFICIENTS IN EQUATIONS (5) AND (6)}

Expanding equation (4) in a Taylor series about the mean values $(\bar{b}, \bar{w})$ yields

$$
\begin{aligned}
r= & r(\bar{b}, \bar{w})+\left[(b-\bar{b}) \frac{\partial r(\bar{b}, \bar{w})}{\partial b}+(w-\bar{w}) \frac{\partial r(\bar{b}, \bar{w})}{\partial w}\right] \\
& +\frac{1}{2 !}\left[(b-\bar{b}) \frac{\partial r(\bar{b}, \bar{w})}{\partial b}+(w-\bar{w}) \frac{\partial r(\bar{b}, \bar{w})}{\partial w}\right]^{2}+\ldots
\end{aligned}
$$

Equation (15) can be truncated to a certain order and thereby the mean and variance of $r$ can be estimated, provided that the corresponding order of moments in $b$ and $w$ are available. However, the estimations in skewness or higher-order statistics require a large sample size, which might not be available for geotechnical data from the site. Thus, the mean and variance in $b$ and $w$ can be employed to predict the mean and variance in $r$. The second-order approximate mean of $r$ is

$$
\begin{aligned}
\bar{r} \approx & r(\bar{b}, \bar{w})+\rho_{b w} \sigma_{b} \sigma_{w} \frac{\partial^{2} r(\bar{b}, \bar{w})}{\partial b \partial w} \\
& +\frac{1}{2} \sigma_{b}^{2} \frac{\partial^{2} r(\bar{b}, \bar{w})}{\partial b^{2}}+\frac{1}{2} \sigma_{w}^{2} \frac{\partial^{2} r(\bar{b}, \bar{w})}{\partial w^{2}}
\end{aligned}
$$

The first-order approximate variance of $r$ is

$$
\begin{aligned}
\operatorname{Var}[r] \approx & \sigma_{b}^{2}\left[\frac{\partial r(\bar{b}, \bar{w})}{\partial b}\right]^{2}+\sigma_{w}^{2}\left[\frac{\partial r(\bar{b}, \bar{w})}{\partial w}\right]^{2} \\
& +2 \rho_{b w} \sigma_{b} \sigma_{w} \frac{\partial r(\bar{b}, \bar{w})}{\partial b} \frac{\partial r(\bar{b}, \bar{w})}{\partial w}
\end{aligned}
$$

where $\sigma_{b}$ and $\sigma_{w}$ are the standard deviations of $b$ and $w$, respectively; $\rho_{b w}$ is the correlation coefficient between $b$ and $w$, of which the value is zero in this study as they are independent random variables. 
Only the first-order approximation for variance is used because the second-order approximation requires the kurtosis in $b$ and $w$, which is usually not available. Thus, equations (16) and (17) can be rewritten as

$$
\begin{aligned}
& \bar{r}=r(\bar{b}, \bar{w})+\frac{\operatorname{Var}[b]}{2} \times V_{1}+\frac{\operatorname{Var}[w]}{2} \times V_{2} \\
& \operatorname{Var}[r]=\operatorname{Var}[b] \times V_{3}+\operatorname{Var}[w] \times V_{4}
\end{aligned}
$$
where $\quad V_{1}=\partial^{2} r(\bar{b}, \bar{w}) / \partial b^{2} ; \quad V_{2}=\partial^{2} r(\bar{b}, \bar{w}) / \partial w^{2} ; \quad V_{3}=$
$[\partial r(\bar{b}, \bar{w}) / \partial b]^{2} ; \quad$ and $V_{4}=[\partial r(\bar{b}, \bar{w}) / \partial w]^{2}$. Those first- and second-order partial differentiation terms can be computed recursively as follows

$$
\begin{aligned}
& \frac{\partial r(\bar{b}, \bar{w})}{\partial b}=\frac{-1}{\bar{A}(\bar{b})^{2}}\left[m-\frac{n \bar{w}}{(\overline{w x}+a)}\right] r(\bar{b}, \bar{w}) \\
& \frac{\partial r(\bar{b}, \bar{w})}{\partial w}=\xi r(\bar{b}, \bar{w})
\end{aligned}
$$

$$
\begin{aligned}
& \frac{\partial^{2} r(\bar{b}, \bar{w})}{\partial b^{2}}=\left\{\frac{-2}{\bar{b}}+\frac{1+a}{(1+\bar{w})(\bar{b})^{2}}\left[-m+\frac{n \bar{w}}{\overline{w x}+a}-\frac{\overline{(\bar{w}}+a)}{}\right.\right. \\
& \frac{\partial^{2} r(\bar{b}, \bar{w})}{\partial w^{2}}=\left\{\frac{2 m \bar{B}}{(1+\bar{w})^{3}}+\frac{n \bar{B}[\bar{B}+2 \bar{w} \bar{B}+2(1+\bar{w}) a]}{\left[\bar{w}(1+\bar{w}) \bar{B}+(1+\bar{w})^{2} a\right]^{2}}+\xi^{2}\right\} r(\bar{b}, \bar{w})
\end{aligned}
$$

in which $\xi=\left[\bar{B} /(1+\bar{w})^{2}\right](-m-\{(1+\bar{w}) n /[\bar{w} \bar{B}+(1+\bar{w}) a]\})$; $\bar{A}=[(1+\bar{w}) /(1+a)] ; \bar{B}=(1+a)[(1 / \bar{b})-1]$.

\section{NOTATION}

a water-cement mass ratio of the cement slurry

$b$ binder mass fraction in deep-mixed column

$\bar{b}$ mean value of binder mass fraction in deep-mixed column

$F_{\mathrm{R}}(r)$ cumulative distribution function of strength ratio

$f\left(\mu_{1}, \mu_{2} ; \tau\right)$ joint probability density function at separation distance $\tau$

$f_{\mathrm{B}}(b)$ marginal probability density function of binder mass fraction

$f_{\mathrm{b}, \mathrm{w}}(b, w)$ joint probability density function between binder mass fraction and in situ water content

$f_{\mathrm{R}}(r)$ probability density function of strength ratio

$f_{\mathrm{W}}(w)$ marginal probability density function of in situ water content

$m$ experimentally fitted coefficients for strength function

$n$ experimentally fitted coefficients for strength function

$q_{0} \quad$ experimentally fitted coefficients for strength function

$q_{\mathrm{u}} \quad$ unconfined compressive strength

$r$ strength ratio

$\bar{r}$ mean value of strength ratio

$r(b, w)$ strength ratio as a function of binder mass fraction and in situ water content

$\boldsymbol{u}_{1}, \boldsymbol{u}_{2}$ location vectors

$V_{1}, V_{2}, V_{3}, V_{4}$ coefficients relating to partial differentiation of strength function

$w$ water content of in situ soil

$\bar{w}$ mean value of water content of in situ soil

$x$ soil-cement mass ratio

$\bar{x}$ mean value of soil-cement mass ratio

$y$ water-cement mass ratio

$\bar{y}$ mean value of water-cement mass ratio

\author{
$\zeta$ autocorrelation function \\ $\theta$ scale of fluctuation \\ $\mu_{1}, \mu_{2}$ location indices \\ $\xi$ differential factor \\ $\rho_{b w} \quad$ coefficient of correlation between binder mass \\ fraction and in situ water content \\ $\sigma_{b} \quad$ standard deviation of binder mass fraction \\ $\sigma_{w}$ standard deviation of in situ water content \\ $\tau$ separation distance
}

\section{REFERENCES}

Akbas, S. O. \& Kulhawy, F. H. (2010). Characterization and estimation of geotechnical variability in Ankara clay: a case history. Geotech. Geol. Engng 28, No. 5, 619-631.

Al-Naqshabandy, M. S., Bergman, N. \& Larsson, S. (2012). Strength variability in lime-cement columns based on cone penetration test data. Proc. Instn Civ. Engrs - Ground Improvement 165, No. 1, 15-26, https://doi.org/10.1680/grim.2012.165.1.15.

Alonso, E. E. \& Krizek, R. J. (1975). Stochastic formulation of soil properties. Proceedings of the 2nd international conference on

applications of statistics and probability in soil and structural engineering, Aachen, Germany, pp. 9-32.

Baecher, B. G. \& Christian, J. T. (2003). Reliability and statistics in geotechnical engineering. Chichester, UK: John Wiley \& Sons.

Bergman, N., Al-Naqshabandy, M. S. \& Larsson, S. (2013). Variability of strength and deformation properties in limecement columns evaluated from CPT and KPS measurements. Georisk 7, No. 1, 21-36.

Bruce, M. E. C., Berg, R. R., Collin, J. G., Filz, G. M., Terashi, M. \& Yang, D. S. (2013). Federal highway administration design manual: deep mixing for embankment and foundation support. McLean, VA, USA: Department of Transportation Federal Highway Administration.

Chen, J., Liu, Y. \& Lee, F. H. (2016). A statistical model for the unconfined compressive strength of deep mixed columns. Géotechnique 66, No. 5, 351-365, https://doi.org/10.1680/jgeot. 14.P.162.

De Souza, Z. M., Junior, J. M. \& Peraira, G. T. (2009). Spatial variability of the physical and mineralogical properties of the soil from the areas with variation in landscape shapes. Braz. Archs Biol. Technol. 52, No. 2, 305-316.

Diaz-Padilla, J. (1974). Settlement of structures on shallow foundations: a probabilistic analysis. $\mathrm{PhD}$ thesis, Massachusetts Institute of Technology, Cambridge, MA, USA.

Filz, G. M. \& Navin, M. P. (2010). A practical method to account for strength variability of deep-mixed ground. In GeoFlorida 2010: advances in analysis, modeling and design (eds D. O. Fratta, A. J. Puppala and B. Muhunthan), GSP 199, pp. 2426-2433. Reston, VA, USA: American Society of Civil Engineers.

Grote, K., Anger, C., Kelly, B., Hubbard, S. \& Rubin, Y. (2010). Characterization of soil water content variability and soil texture using GPR groundwave techniques. J. Environ. Engng Geophys. 15, No. 3, 93-110.

Honjo, Y. (1982). A probabilistic approach to evaluate shear strength of heterogeneous stabilized ground by deep mixing method. Soils Found. 22, No. 1, 23-38.

Larsson, S. \& Nilsson, A. (2009). Horizontal strength variability in lime-cement columns - a field study. In Proceedings of international symposium on deep mixing and admixture stabilization (ed. M. Kitazume), pp. 629-634. Okinawa, Japan: Port and Airport Research Institute (PARI).

Larsson, S., Stille, H. \& Olsson, L. (2005). On horizontal variability in lime-cement columns in deep mixing. Géotechnique 55, No. 1, 33-44, https://doi.org/10.1680/geot. 2005.55.1.33. 
Lee, F. H., Lee, C. H. \& Dasari, G. R. (2006). Centrifuge modeling of wet deep mixing processes in soft clays. Géotechnique 56, No. 10, 677-691, https://doi.org/10.1680/ geot.2006.56.10.677.

Lee, F. H., Lee, C. H., Dasari, G. R. \& Zheng, J. J. (2008). Centrifuge study on uniformity of wet deep mixing. Int. J. Phys. Modelling Geotech. 8, No. 1, 1-20.

Li, D. Q., Xiao, T., Cao, Z. J., Zhou, C. B. \& Zhang, L. M. (2016). Enhancement of random finite element method in reliability analysis and risk assessment of soil slopes using Subset Simulation. Landslides 13, No. 2, 293-303.

Liu, Y., Lee, F. H., Quek, S. T. \& Beer, M. (2014). Modified linear estimation method for generating multi-dimensional multivariate Gaussian field in modelling material properties. Probabilistic Engng Mech. 38, 42-53.

Liu, Y., Lee, F. H., Quek, S. T., Chen, J. E. \& Yi, J. T. (2015). Effect of spatial variation of strength and modulus on the lateral compression response of cement-admixed clay slab. Géotechnique 65, No. 10, 851-865, https://doi.org/10.1680/ jgeot.14.P.254

Liu, Y., Jiang, Y., Xiao, H. \& Lee, F. H. (2017). Determination of representative strength of deep cement-mixed clay from core strength data. Géotechnique 67, No. 4, 350-364, https://doi.org/10.1680/jgeot.16.P.105.

Namikawa, T. (2015). Conditional probabilistic analysis of cementtreated soil column strength. Int. J. Geomech., ASCE 16, No. 1, paper no. 04015021, https://doi.org/10.1061/(ASCE)GM. 1943-5622.0000481.
Namikawa, T. \& Koseki, J. (2013). Effects of spatial correlation on compression behavior of cement-treated column. J. Geotech. Geoenviron. Engng, ASCE 139, No. 8, 1346-1359.

Navin, M. P. (2005). Stability of embankments founded on soft soil improved with deep-mixing-method columns. $\mathrm{PhD}$ thesis, Virginia Polytechnic Institute and State University, Blacksburg, VA, USA.

Phoon, K. K. \& Kulhawy, F. H. (1999). Characterization of geotechnical variability. Can. Geotech. J. 36, No. 4, 612-624.

Tang, X. S., Li, D. Q., Zhou, C. B. \& Phoon, K. K. (2015). Copula-based approaches for evaluating slope reliability under incomplete probability information. Struct. Saf. 52, 90-99.

Teo, H. J. (2017). 1-G modelling of wet deep mixing with fibres. $\mathrm{PhD}$ thesis, National University of Singapore, Singapore.

Tsuchida, T. \& Tang, Y. X. (2015). Estimation of compressive strength of cement-treated marine clays with different initial water contents. Soils Found. 55, No. 2, 359-374.

Vanmarcke, E. H. (1983). Random fields: analysis and synthesis. Cambridge, MA, USA: MIT Press.

Western, A. W. \& Grayson, R. B. (1998). The Tarrawarra data set: soil moisture patterns, soil characteristics, and hydrological flux measurements. Wat. Resour. Res. 34, No. 10, 2765-2768.

Xiao, H., Lee, F. H. \& Chin, K. G. (2014). Yielding of cementtreated marine clay. Soils Found. 54, No. 3, 488-501.

Zhang, D. M., Gu, X. L., Yu, Q. Q., Huang, H. W., Wan, B. L. \& Jiang, C. (2018). Full probabilistic analysis of FRP-to-concrete bonded joints considering model uncertainty. Composite Structs 185, 786-806. 